Proceedings

\title{
New 1,3,5-Triazine Derivatives Incorporating Aminobenzenesulfonamide, Aminoalcohol, Piperazine, Chalcone or Stilbene Structural Motifs and Evaluation of Their Antioxidative Activity ${ }^{\dagger}$
}

\author{
Eva Havránková ${ }^{1, *}{ }^{,}$, Nikola Čalkovská ${ }^{1}$, Tereza Padrtová ${ }^{1}$, Jozef Csöllei ${ }^{1}$, Radka Opatřilová ${ }^{1}$ \\ and Pavel Pazdera ${ }^{2}$ \\ 1 Department of Chemical Drugs, Faculty of Pharmacy, University of Veterinary and Pharmaceutical \\ Sciences, 61242 Brno, Czech Republic; F16222@vfu.cz (N.Č); padrtovat@vfu.cz (T.P.); csolleij@vfu.cz (J.C.); \\ opatrilovar@vfu.cz (R.O.); \\ 2 Department of Chemistry, Faculty of Science, Centre for Syntheses at Sustainable Conditions and Their \\ Management, Masaryk University, 62500 Brno, Czech Republic; pazdera@chemi.muni.cz \\ * Correspondence: havrankovae@vfu.cz \\ + Presented at the 23rd International Electronic Conference on Synthetic Organic Chemistry, 15 November- \\ 15 December 2019. Available online: https://ecsoc-23.sciforum.net/.
}

Published: 14 January 2020

\begin{abstract}
A series of 1,3,5-triazine derivatives, incorporating aminobenzenesulfonamide, aminoalcohol/phenol, piperazine, chalcone, or stilbene structural motifs, were evaluated as potential antioxidants. The compounds were prepared by using step by step nucleophilic substitution of chlorine atoms in starting 2,4,6-trichloro-1,3,5-triazine. Reactions were catalyzed by $\mathrm{Cu}(\mathrm{I})$-supported on a weakly acidic resin. The radical scavenging activity was determined in terms of \%inhibition activity and IC50, using the ABTS method. Trolox and ascorbic acid (ASA) were used as standards. In the lowest used concentration $1 \times 10^{-4} \mathrm{M}$, the \%inhibition activity at time 0 min was comparable with both standards at least for 10 compounds. After 60 min compounds 1, 2, 9 and 25 showed nearly twice \%inhibition (73.44-87.09\%) in comparison with standards (Trolox $=41.49 \%$; ASA $=31.07 \%$ ). Values of IC 50 correlated with \%inhibition activity. For compounds 1, 2, 9 and 25 values of $\mathrm{IC}_{50}$ in time $60 \mathrm{~min}(17.16-27.78 \mu \mathrm{M})$ were 5 times lower than IC 50 of both standards (Trolox $=178.33 \mu \mathrm{M} ; \mathrm{ASA}=147.47 \mu \mathrm{M})$. Based on these results, the presented 1,3,5-triazine derivatives and their analogs have a high potential in the treatment of illnesses caused or related to oxidative stress.
\end{abstract}

Keywords: 1,3,5-triazine; 4-aminophenol; hydroxychalcone; hydroxystilbene; antioxidative activity; ABTS method

\section{Introduction}

Oxidative stress or damage is the imbalance between the capacity of antioxidative protection systems of the organism and the occurrence of reactive oxygen species and/or reactive nitrogen species [1,2]. Oxygen reactive species, such as free radicals, can be produced by metabolic pathways, UV irradiation, environmental pollutants, and others [1-6].

Damage of cells caused by oxidative stress is correlated to pathophysiology and pathogenesis of a wide range of diseases, i.e., Alzheimer's, Huntington's, and Parkinson's diseases, inflammatory diseases, cardiovascular diseases, diabetes, and cancer [1,3,4,7-9].

The structure of molecules with antioxidant activity can be very diverse. Recently, triazine derivatives with desirable antioxidant activity were described. Gonzalez et al. proved the antioxidant activity of their 1,3,5-triazine bridged small molecules (Figure 1a) [3]. 5,6-Diphenyl-3-oxo-1,2,4- 
triazine linked piperazine derivatives (Figure 1b) [10] or arylidene hydrazine derivatives of substituted 1,2,4-triazine scaffolds also exhibit attractive radical scavenging effects (Figure 1c) [11]. Substituted triazene benzenesulfonamides (Figure 1d), synthesized by Akocak et al., also showed significant antioxidative activity [12]. A promising antioxidant effects can be found even between chalcone derivatives. For example, Nageshi et al. synthesized very effective antioxidants based on the structure of chalcones and containing phenolic functional group (Figure 1e) [13]. Phenolic compounds are one of the largest groups of antioxidants with high activity $[1,4,7-9,14]$. An example of phenolic antioxidant, which is used in medicine as an adjuvant treatment of cardiovascular diseases, metabolic syndrome, type 2 diabetes, and inflammatory diseases, is resveratrol (stilbene derivative, Figure 1f) [4].

Antioxidants possess great potential as drugs suitable for the treatment of various diseases related to oxidative stress. Their structural diversity provides many possibilities for the design and synthesis of new highly effective agents $[1,2,4]$.

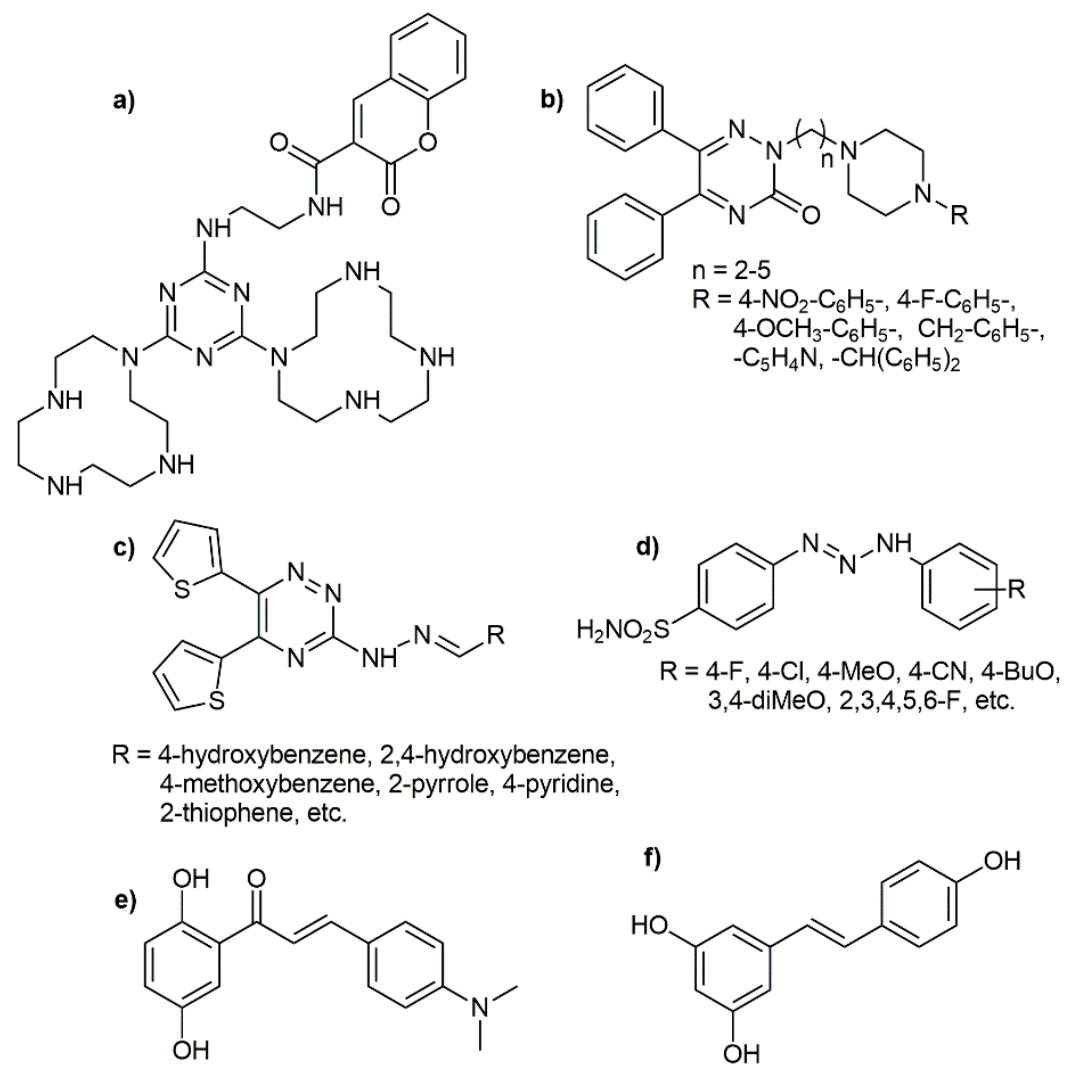

Figure 1. Examples of diversity of antioxidants with high activity: (a) 1,3,5-triazine bridged small molecules [3]; (b) biphenyl-3-oxo-1,2,4-triazine linked piperazine derivatives [10]; (c) arylidene hydrazine derivatives of substituted 1,2,4-triazine [11]; (d) substituted aminobenzenesulfonamides [12]; (e) chalcone derivatives [13]; (f) stilbene derivatives (resveratrol) [4].

\section{Materials and Methods}

\subsection{Chemistry}

Target compounds were prepared according to the methodology published in [15] by step by step nucleophile substitution of chlorine atoms in starting 2,4,6-trichloro-1,3,5-triazine. The appropriate starting compound reacted with a nucleophile and anhydrous potassium carbonate in a molar ratio 1:1:1. Reactions were catalyzed by $\mathrm{Cu}(\mathrm{I})$-supported on a weakly acidic resin. The substitution of first, second, or third chlorine atom was controlled by the temperature mode (Scheme 1). 


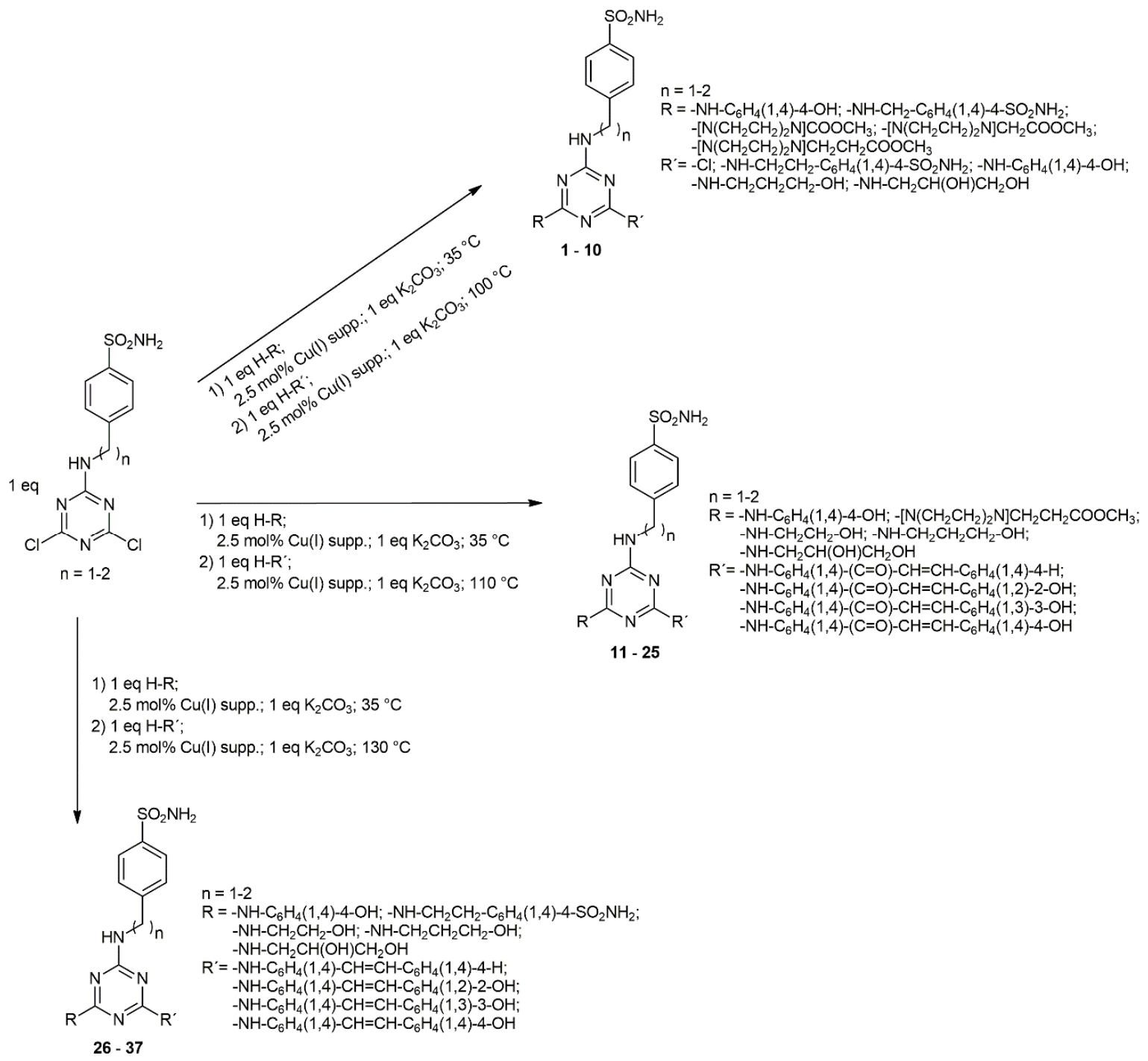

Scheme 1. General synthetic scheme of target compounds.

\subsection{Characterization of New Compounds}

The characterization of only two representative compounds is given here. The full characterization of all new compounds will be part of the published full manuscript.

(E)-4-[2-(\{4-[(2-Hydroxyethyl)amino]-6-(\{4-[3-(3-hydroxyphenyl)acryloyl]phenyl\}amino)-1,3,5-triazin-2-

yllamino)ethyllbenzenesulfonamide (19): 77.7\%; brown solid; m.p. 256-258 ${ }^{\circ} \mathrm{C} ;{ }^{1} \mathrm{H}-\mathrm{NMR}(500 \mathrm{MHz}$, DMSO- $\left.d_{6}\right) \delta$ ppm $7.91(2 \mathrm{H}, \mathrm{d}, J=8.1 \mathrm{~Hz}, \mathrm{CH}), 7.80(2 \mathrm{H}, \mathrm{d}, J=8.1 \mathrm{~Hz}, \mathrm{CH}), 7.71(1 \mathrm{H}, \mathrm{d}, J=15.5 \mathrm{~Hz}$, $\underline{\mathrm{CH}}=\mathrm{CH}-\mathrm{CO}), 7.56(2 \mathrm{H}, \mathrm{d}, J=8.1 \mathrm{~Hz}, \mathrm{CH}), 7.48(1 \mathrm{H}, \mathrm{d}, J=15.5 \mathrm{~Hz}, \mathrm{CH}=\underline{\mathrm{CH}}-\mathrm{CO}), 7.26(2 \mathrm{H}, \mathrm{d}, J=8.1$ $\mathrm{Hz}, \mathrm{CH}), 6.91(2 \mathrm{H}, \mathrm{d}, J=8.1 \mathrm{~Hz}, \mathrm{CH}), 6.69\left(7 \mathrm{H}, \mathrm{s}, \mathrm{OH}, \mathrm{NH}, \mathrm{NH}_{2}\right), 4.56-4.52\left(2 \mathrm{H}, \mathrm{m}, \mathrm{CH}_{2}\right), 3.55-3.53$ (2H, m, $\left.\underline{\mathrm{C}}_{2}-\mathrm{OH}\right), 3.34-3.32\left(2 \mathrm{H}, \mathrm{m}, \mathrm{NH}-\underline{\mathrm{H}}_{2}\right), 2.93-2.91\left(2 \mathrm{H}, \mathrm{m}, \mathrm{CH}_{2}\right)$; ${ }^{13} \mathrm{C}-\mathrm{NMR}$ (125 MHz, DMSO$\left.d_{6}\right) \delta$ ppm 189.4, 168.6, 166.3, 165.6, 146.7, 144.5, 144.1, 142.3, 141.8, 141.2, 138.2, 130.4, 128.2, 126.6, 125.9, 119.7, 118.6, 114.2, 58.8, 44.5, 41.9, 36.6; IR $v_{\max }\left(\mathrm{cm}^{-1}\right)$ 3336, $3222\left(\mathrm{OH}, \mathrm{NH}, \mathrm{NH}_{2}\right), 2930\left(\mathrm{CH}_{\text {alif, }}\right.$ $\mathrm{CH}_{2}$ alif), 1698, 1660, 1654, 1635 (C=C, C=N, C=O), 1583, 1345, 1155, $\left(\mathrm{SO}_{2} \mathrm{NH}_{2}\right), 1076(\mathrm{C}-\mathrm{OH}), 1034$ (COC)

(E)-Methyl 3-(4-\{4-(\{4-[3-(4-hydroxyphenyl) acryloyl]phenyl\}amino)-6-[(4-sulfamoyl-phenethyl)amino] 1,3,5-triazin-2-ylspiperazin-1-yl)propanoate (25): 78.1\%; orange solid; m.p. 101-103 ${ }^{\circ} \mathrm{C} ;{ }^{1} \mathrm{H}-\mathrm{NMR}(500$ MHz, DMSO- $\left.d_{6}\right) \delta$ ppm $7.97(2 \mathrm{H}, \mathrm{d}, J=8.1 \mathrm{~Hz}, \mathrm{CH}), 7.94(2 \mathrm{H}, \mathrm{d}, J=8.1 \mathrm{~Hz}, \mathrm{CH}), 7.8(1 \mathrm{H}, \mathrm{d}, J=15.5$ $\mathrm{Hz}, \underline{\mathrm{CH}}=\mathrm{CH}-\mathrm{CO}), 7.42(1 \mathrm{H}, \mathrm{d}, J=15.5 \mathrm{~Hz}, \mathrm{CH}=\underline{\mathrm{CH}}-\mathrm{CO}), 7.31(2 \mathrm{H}, \mathrm{d}, J=8.1 \mathrm{~Hz}, \mathrm{CH}), 7.28(2 \mathrm{H}, \mathrm{d}, J=$ $8.1 \mathrm{~Hz}, \mathrm{CH}), 6.99(2 \mathrm{H}, \mathrm{d}, J=8.1 \mathrm{~Hz}, \mathrm{CH}), 6.68(2 \mathrm{H}, \mathrm{d}, J=8.1 \mathrm{~Hz}, \mathrm{CH}), 3.91-3.86\left(4 \mathrm{H}, \mathrm{m}, \mathrm{CH}_{2}\right), 3.74-$ $3.71\left(2 \mathrm{H}, \mathrm{m}, \mathrm{NH}-\mathrm{C}_{2}\right), 3.65\left(3 \mathrm{H}, \mathrm{s}, \mathrm{CH}_{3}\right), 2.99-2.87\left(4 \mathrm{H}, \mathrm{m}, \mathrm{CH}_{2}\right), 2.76-2.74\left(2 \mathrm{H}, \mathrm{m}, \mathrm{CH}_{2}\right), 2.59(2 \mathrm{H}, \mathrm{t}, J$ 
$\left.=7.8 \mathrm{~Hz}, \mathrm{~N}-\mathrm{CH}_{2}\right), 2.11\left(2 \mathrm{H}, \mathrm{t}, J=7.8 \mathrm{~Hz}, \mathrm{CH}_{2}-\mathrm{COO}\right) ;{ }^{13} \mathrm{C}-\mathrm{NMR}\left(125 \mathrm{MHz}, \mathrm{DMSO}-d_{6}\right) \delta \mathrm{ppm}$ 186.7, 171.9 , 167.8, 167.1, 162.6, 159.9, 158.4, 154.2, 150.9, 143.8, 142.7, 144.3, 119.2, 131.3, 130.6, 126.8, 124.9, 116.3, 113.1, 66.4, 51.3, 51.1, 44.3, 43.7, 35.1, 31.6; IR $v_{\max }\left(\mathrm{cm}^{-1}\right) 3332,3218\left(\mathrm{OH}, \mathrm{NH}, \mathrm{NH}_{2}\right), 2950$ ( $\left.\mathrm{CH}_{\text {alif, }} \mathrm{CH}_{2 a l i f}\right)$ $1698,1683,1669,1651,1646,1630,1588(\mathrm{C}=\mathrm{C}, \mathrm{C}=\mathrm{N}, \mathrm{COO}), 1349\left(\mathrm{SO}_{2} \mathrm{NH}_{2}\right), 1227(\mathrm{OH}), 1162\left(\mathrm{SO}_{2} \mathrm{NH}_{2}\right)$, 1105,1028 (COC)

\subsection{Determination of Antioxidant Activity by ABTS Method}

BioTekTM CytationTM 3 Cell Imaging Multi-Mode Reader (BioTek USA) equipment was used for measurement.

A stock solution containing free cation radicals $\mathrm{ABTS}^{+}$was prepared as follows: $10 \mathrm{~mL}$ of an aqueous solution of ABTS $(7.4 \mathrm{mmol})$ and $10 \mathrm{~mL}$ of an aqueous solution of potassium peroxodisulfate $(2.6 \mathrm{mmol})$ were mixed and allowed to stand at room temperature in the dark for $24 \mathrm{~h}$. After the end of the incubation period, a $50 \mathrm{~mL}$ volumetric flask was charged with $1.1 \mathrm{~mL}$ of ABTS stock solution and filled up with ethanol. This solution of $\mathrm{ABTS}^{+}$was used for the determination of antioxidant activity.

Tested compounds were dissolved in DMSO. Solutions of concentration $1 \times 10^{-2} \mathrm{M}, 1 \times 10^{-3} \mathrm{M}, 5$ $\times 10^{-4} \mathrm{M}, 1 \times 10^{-4} \mathrm{M}, 5 \times 10^{-5} \mathrm{M}, 1 \times 10^{-5} \mathrm{M}$ and $1 \times 10^{-6} \mathrm{M}$ were prepared.

Antioxidant activity of tested compounds was determined as follows: $200 \mu \mathrm{L}$ of a solution of $\mathrm{ABTS}^{+}$and $10 \mu \mathrm{L}$ of a solution of the tested compound of appropriate concentration were mixed. Antioxidant activity was determined by measurement of absorbance at $754 \mathrm{~nm}$ (max absorbance of $\mathrm{ABTS}^{+}$) after 0, 5, 30, and $60 \mathrm{~min}$. Pure DMSO was used as a blank. Trolox and ascorbic acid were used as standards (solutions were prepared at the same concentrations as tested compounds).

ABTS cation radical scavenging ability of tested compounds expressed as \%inhibition was calculated using the following Equation (1) [14]:

$$
\%=\frac{A_{B}-A}{A_{B}} 100
$$

where: $A_{B}$ means absorbance of blank; $A$ means absorbance of the sample.

The values of IC ${ }_{50}$ were determined as follows: The values of \%inhibition of $\mathrm{ABTS}^{+}$radical in time (0 min, $5 \mathrm{~min}, 30 \mathrm{~min}$, and $60 \mathrm{~min}$ ) at concentrations of $1 \times 10^{-2} \mathrm{M}, 1 \times 10^{-3} \mathrm{M}, 5 \times 10^{-4} \mathrm{M}, 1 \times 10^{-4}$ $\mathrm{M}, 5 \times 10^{-5} \mathrm{M}, 1 \times 10^{-5} \mathrm{M}$, and $1 \times 10^{-6} \mathrm{M}$ were determined; a plot of concentration versus \%inhibition was obtained from the data; a number 50 was substituted for the unknown $\times$ ( $50 \%$ inhibition) in the trendline equation, and value $\mathrm{IC}_{50}$ was calculated.

All calculated results are shown in Tables 1 and 2.

\section{Results and Discussion}

In this paper, a series of 1,3,5-triazine derivatives, incorporating aminobenzenesulfonamide, aminoalcohol/phenol, piperazine, chalcone, or stilbene structural motifs were evaluated as potential antioxidants. These 1,3,5-derivatives were primarily designed as inhibitors of hCA IX (isozyme of human carbonic anhydrase), and the activity of this enzyme is correlated with tumor growth. Since the synthesized compounds contain one or more structural motifs in their structure that already demonstrated antioxidant properties (Section 1), the screening of their antioxidant activity was made. The ABTS assay was used for the evaluation of an antioxidant activity. The results were compared to commercially available standards Trolox and ascorbic acid. The ABTS method was performed because tested compounds contain either 4-aminophenol or hydroxystilbene or hydroxychalcone structural moieties. Currently, the ABTS method is one of the most widely used for the determination of the antioxidative properties of phenols [14]. We assumed that the mechanism of action is probably very similar to resveratrol and analogous compounds.

We were pleased to find that almost half of the tested compounds had exhibited excellent antioxidative activity. The results of the (cation)radical scavenging activity are shown in Table 1 (\%inhibition activity) and Table 2 (values of IC50). 
Table 1. ABTS cation radical scavenging ability of tested compounds expressed as \% inhibition in different times ( 0 min, 5 min, 30 min, and 60 min) and different concentrations $\left(1 \times 10^{-2} \mathrm{M}\right.$ and $\left.1 \times 10^{-4} \mathrm{M}\right)$. Significant results are shown in bold.

\begin{tabular}{|c|c|c|c|c|c|c|c|c|c|c|c|}
\hline \multirow{2}{*}{ Compound } & \multirow{2}{*}{$\mathbf{n}$} & \multirow{2}{*}{$\mathbf{R}_{1}$} & \multirow{2}{*}{$\mathbf{R}_{2}$} & \multicolumn{4}{|c|}{$1 \times 10^{-2} \mathrm{M} ;(\%)^{2}$} & \multicolumn{4}{|c|}{$1 \times 10^{-4} \mathrm{M} ;(\%)^{2}$} \\
\hline & & & & $0 \mathrm{~min}$ & $5 \mathrm{~min}$ & $30 \mathrm{~min}$ & $60 \mathrm{~min}$ & $0 \mathrm{~min}$ & $5 \mathrm{~min}$ & $30 \mathrm{~min}$ & $60 \mathrm{~min}$ \\
\hline $\mathbf{1}^{1}$ & 1 & $\mathrm{NH}-\mathrm{C}_{6} \mathrm{H}_{4}(1,4)-4-\mathrm{OH}$ & $\mathrm{Cl}$ & 92.05 & 92.00 & 91.88 & 91.88 & 31.52 & 38.51 & 44.71 & 73.51 \\
\hline $2^{1}$ & 2 & $\mathrm{NH}-\mathrm{C}_{6} \mathrm{H}_{4}(1,4)-4-\mathrm{OH}$ & $\mathrm{Cl}$ & 91.38 & 91.26 & 91.21 & 91.21 & 45.38 & 49.84 & 63.65 & 73.44 \\
\hline $3^{1}$ & 1 & $\mathrm{NH}-\mathrm{CH}_{2}-\mathrm{C}_{6} \mathrm{H}_{4}(1,4)-4-\mathrm{SO}_{2} \mathrm{NH}_{2}$ & $\mathrm{NH}-\mathrm{CH}_{2} \mathrm{CH}_{2}-\mathrm{C}_{6} \mathrm{H}_{4}(1,4)-4-\mathrm{SO}_{2} \mathrm{NH}_{2}$ & 35.58 & 47.98 & 58.35 & 66.69 & 21.82 & 24.81 & 27.23 & 35.57 \\
\hline $4^{1}$ & 2 & $\mathrm{NH}-\mathrm{CH}_{2}-\mathrm{C}_{6} \mathrm{H}_{4}(1,4)-4-\mathrm{SO}_{2} \mathrm{NH}_{2}$ & $\mathrm{NH}-\mathrm{CH}_{2} \mathrm{CH}_{2}-\mathrm{C}_{6} \mathrm{H}_{4}(1,4)-4-\mathrm{SO}_{2} \mathrm{NH}_{2}$ & 51.53 & 74.64 & 79.54 & 90.36 & 31.46 & 35.24 & 40.99 & 41.83 \\
\hline $5^{1}$ & 1 & {$\left[\mathrm{~N}\left(\mathrm{CH}_{2} \mathrm{CH}_{2}\right)_{2} \mathrm{~N}\right] \mathrm{COOCH}_{3}$} & $\mathrm{NH}-\mathrm{CH}_{2} \mathrm{CH}(\mathrm{OH}) \mathrm{CH}_{2} \mathrm{OH}$ & 90.70 & 92.84 & 92.95 & 92.90 & 24.02 & 31.01 & 39.92 & 52.99 \\
\hline $6^{1}$ & 1 & {$\left[\mathrm{~N}\left(\mathrm{CH}_{2} \mathrm{CH}_{2}\right)_{2} \mathrm{~N}\right] \mathrm{CH}_{2} \mathrm{COOCH}_{3}$} & $\mathrm{NH}-\mathrm{CH}_{2} \mathrm{CH}(\mathrm{OH}) \mathrm{CH}_{2} \mathrm{OH}$ & 63.98 & 82.13 & 87.15 & 91.77 & 25.37 & 35.07 & 34.73 & 50.06 \\
\hline $7^{1}$ & 1 & {$\left[\mathrm{~N}\left(\mathrm{CH}_{2} \mathrm{CH}_{2}\right)_{2} \mathrm{~N}\right] \mathrm{CH}_{2} \mathrm{COOCH}_{3}$} & $\mathrm{NH}-\mathrm{CH}_{2} \mathrm{CH}_{2}-\mathrm{C}_{6} \mathrm{H}_{4}(1,4)-4-\mathrm{SO}_{2} \mathrm{NH}_{2}$ & 79.20 & 86.47 & 88.33 & 88.39 & 24.81 & 34.28 & 42.90 & 64.38 \\
\hline $8^{1}$ & 1 & {$\left[\mathrm{~N}\left(\mathrm{CH}_{2} \mathrm{CH}_{2}\right)_{2} \mathrm{~N}\right] \mathrm{CH}_{2} \mathrm{CH}_{2} \mathrm{COOCH}_{3}$} & $\mathrm{NH}_{-} \mathrm{C}_{6} \mathrm{H}_{4}(1,4)-4-\mathrm{OH}$ & 89.63 & 89.69 & 89.69 & 89.69 & 38.28 & 39.41 & 41.89 & 60.66 \\
\hline $9^{1}$ & 1 & $\mathrm{NH}-\mathrm{C}_{6} \mathrm{H}_{4}(1,4)-4-\mathrm{OH}$ & $\mathrm{NH}-\mathrm{CH}_{2} \mathrm{CH}(\mathrm{OH}) \mathrm{CH}_{2} \mathrm{OH}$ & 89.40 & 89.29 & 89.18 & 89.12 & 33.55 & 36.31 & 48.71 & 78.24 \\
\hline $10^{1}$ & 1 & $\mathrm{NH}-\mathrm{CH}_{2}-\mathrm{C}_{6} \mathrm{H}_{4}(1,4)-4-\mathrm{SO}_{2} \mathrm{NH}_{2}$ & $\mathrm{NH}-\mathrm{CH}_{2} \mathrm{CH}_{2} \mathrm{CH}_{2} \mathrm{OH}$ & 43.81 & 62.80 & 75.54 & 82.19 & 26.39 & 29.09 & 30.67 & 49.16 \\
\hline 11 & 1 & $\mathrm{NH}-\mathrm{CH}_{2} \mathrm{CH}_{2} \mathrm{CH}_{2} \mathrm{OH}$ & $-\mathrm{NH}-\mathrm{C}_{6} \mathrm{H}_{4}(1,4)-(\mathrm{C}=\mathrm{O})-\mathrm{CH}=\mathrm{CH}-\mathrm{C}_{6} \mathrm{H}_{4}(1,4)-4-\mathrm{H}$ & 52.03 & 68.04 & 75.88 & 81.57 & 24.36 & 23.68 & 25.88 & 42.62 \\
\hline 12 & 2 & $\mathrm{NH}-\mathrm{CH}_{2} \mathrm{CH}_{2} \mathrm{CH}_{2} \mathrm{OH}$ & $-\mathrm{NH}-\mathrm{C}_{6} \mathrm{H}_{4}(1,4)-(\mathrm{C}=\mathrm{O})-\mathrm{CH}=\mathrm{CH}-\mathrm{C}_{6} \mathrm{H}_{4}(1,4)-4-\mathrm{H}$ & 52.60 & 70.01 & 85.29 & 89.12 & 18.33 & 23.97 & 31.63 & 46.34 \\
\hline 13 & 1 & $\mathrm{NH}-\mathrm{CH}_{2} \mathrm{CH}_{2} \mathrm{CH}_{2} \mathrm{OH}$ & $-\mathrm{NH}-\mathrm{C}_{6} \mathrm{H}_{4}(1,4)-(\mathrm{C}=\mathrm{O})-\mathrm{CH}=\mathrm{CH}-\mathrm{C}_{6} \mathrm{H}_{4}(1,4)-3-\mathrm{OH}$ & 89.57 & 89.57 & 89.63 & 89.63 & 24.70 & 31.91 & 34.62 & 50.79 \\
\hline 14 & 2 & $\mathrm{NH}-\mathrm{CH}_{2} \mathrm{CH}_{2} \mathrm{CH}_{2} \mathrm{OH}$ & $-\mathrm{NH}-\mathrm{C}_{6} \mathrm{H}_{4}(1,4)-(\mathrm{C}=\mathrm{O})-\mathrm{CH}=\mathrm{CH}-\mathrm{C}_{6} \mathrm{H}_{4}(1,4)-3-\mathrm{OH}$ & 90.47 & 90.59 & 90.25 & 90.14 & 19.01 & 18.55 & 23.18 & 38.39 \\
\hline 15 & 1 & $\mathrm{NH}-\mathrm{CH}_{2} \mathrm{CH}_{2} \mathrm{CH}_{2} \mathrm{OH}$ & $-\mathrm{NH}-\mathrm{C}_{6} \mathrm{H}_{4}(1,4)-(\mathrm{C}=\mathrm{O})-\mathrm{CH}=\mathrm{CH}-\mathrm{C}_{6} \mathrm{H}_{4}(1,4)-2-\mathrm{OH}$ & 90.70 & 90.36 & 89.97 & 89.74 & 34.28 & 35.41 & 37.32 & 57.56 \\
\hline 16 & 2 & $\mathrm{NH}-\mathrm{CH}_{2} \mathrm{CH}_{2} \mathrm{CH}_{2} \mathrm{OH}$ & $-\mathrm{NH}-\mathrm{C}_{6} \mathrm{H}_{4}(1,4)-(\mathrm{C}=\mathrm{O})-\mathrm{CH}=\mathrm{CH}-\mathrm{C}_{6} \mathrm{H}_{4}(1,4)-2-\mathrm{OH}$ & 91.43 & 91.32 & 91.04 & 90.76 & 23.46 & 25.04 & 24.13 & 40.03 \\
\hline 17 & 1 & $\mathrm{NH}-\mathrm{CH}_{2} \mathrm{CH}_{2} \mathrm{OH}$ & $-\mathrm{NH}-\mathrm{C}_{6} \mathrm{H}_{4}(1,4)-(\mathrm{C}=\mathrm{O})-\mathrm{CH}=\mathrm{CH}-\mathrm{C}_{6} \mathrm{H}_{4}(1,4)-2-\mathrm{OH}$ & 90.59 & 90.19 & 89.80 & 89.52 & 24.02 & 28.87 & 30.90 & 49.39 \\
\hline 18 & 2 & $\mathrm{NH}-\mathrm{CH}_{2} \mathrm{CH}_{2} \mathrm{OH}$ & $-\mathrm{NH}-\mathrm{C}_{6} \mathrm{H}_{4}(1,4)-(\mathrm{C}=\mathrm{O})-\mathrm{CH}=\mathrm{CH}-\mathrm{C}_{6} \mathrm{H}_{4}(1,4)-2-\mathrm{OH}$ & 90.08 & 89.40 & 88.84 & 89.07 & 22.50 & 23.63 & 24.70 & 47.07 \\
\hline 19 & 2 & $\mathrm{NH}-\mathrm{CH}_{2} \mathrm{CH}_{2} \mathrm{OH}$ & $-\mathrm{NH}-\mathrm{C}_{6} \mathrm{H}_{4}(1,4)-(\mathrm{C}=\mathrm{O})-\mathrm{CH}=\mathrm{CH}-\mathrm{C}_{6} \mathrm{H}_{4}(1,4)-3-\mathrm{OH}$ & 89.12 & 89.01 & 89.01 & 89.12 & 25.49 & 34.79 & 47.81 & 65.28 \\
\hline 20 & 2 & $\mathrm{NH}-\mathrm{CH}_{2} \mathrm{CH}(\mathrm{OH}) \mathrm{CH}_{2} \mathrm{OH}$ & $-\mathrm{NH}-\mathrm{C}_{6} \mathrm{H}_{4}(1,4)-(\mathrm{C}=\mathrm{O})-\mathrm{CH}=\mathrm{CH}-\mathrm{C}_{6} \mathrm{H}_{4}(1,4)-2-\mathrm{OH}$ & 91.15 & 91.04 & 90.76 & 90.53 & 36.25 & 38.85 & 40.59 & 58.97 \\
\hline 21 & 2 & $\mathrm{NH}-\mathrm{C}_{6} \mathrm{H}_{4}(1,4)-4-\mathrm{OH}$ & $-\mathrm{NH}-\mathrm{C}_{6} \mathrm{H}_{4}(1,4)-(\mathrm{C}=\mathrm{O})-\mathrm{CH}=\mathrm{CH}-\mathrm{C}_{6} \mathrm{H}_{4}(1,4)-4-\mathrm{H}$ & 91.21 & 91.15 & 91.15 & 91.09 & 22.56 & 22.61 & 21.32 & 39.07 \\
\hline 22 & 2 & $\mathrm{NH}-\mathrm{C}_{6} \mathrm{H}_{4}(1,4)-4-\mathrm{OH}$ & $-\mathrm{NH}-\mathrm{C}_{6} \mathrm{H}_{4}(1,4)-(\mathrm{C}=\mathrm{O})-\mathrm{CH}=\mathrm{CH}-\mathrm{C}_{6} \mathrm{H}_{4}(1,4)-4-\mathrm{OH}$ & 89.85 & 88.61 & 89.23 & 90.76 & 22.22 & 22.89 & 23.46 & 42.73 \\
\hline 23 & 2 & $\mathrm{NH}-\mathrm{C}_{6} \mathrm{H}_{4}(1,4)-4-\mathrm{OH}$ & $-\mathrm{NH}-\mathrm{C}_{6} \mathrm{H}_{4}(1,4)-(\mathrm{C}=\mathrm{O})-\mathrm{CH}=\mathrm{CH}-\mathrm{C}_{6} \mathrm{H}_{4}(1,4)-3-\mathrm{OH}$ & 90.53 & 90.42 & 90.31 & 90.31 & 25.60 & 27.57 & 32.31 & 36.31 \\
\hline 24 & 2 & $\mathrm{NH}-\mathrm{C}_{6} \mathrm{H}_{4}(1,4)-4-\mathrm{OH}$ & $-\mathrm{NH}-\mathrm{C}_{6} \mathrm{H}_{4}(1,4)-(\mathrm{C}=\mathrm{O})-\mathrm{CH}=\mathrm{CH}-\mathrm{C}_{6} \mathrm{H}_{4}(1,4)-2-\mathrm{OH}$ & 89.40 & 87.32 & 88.56 & 90.19 & 37.72 & 39.47 & 42.28 & 69.34 \\
\hline 25 & 2 & {$\left[\mathrm{~N}\left(\mathrm{CH}_{2} \mathrm{CH}_{2}\right)_{2} \mathrm{~N}\right] \mathrm{CH}_{2} \mathrm{CH}_{2} \mathrm{COOCH}_{3}$} & $-\mathrm{NH}-\mathrm{C}_{6} \mathrm{H}_{4}(1,4)-(\mathrm{C}=\mathrm{O})-\mathrm{CH}=\mathrm{CH}-\mathrm{C}_{6} \mathrm{H}_{4}(1,4)-4-\mathrm{OH}$ & 90.47 & 91.83 & 91.77 & 92.11 & 40.03 & 46.96 & 55.25 & 87.09 \\
\hline 26 & 1 & $\mathrm{NH}-\mathrm{CH}_{2} \mathrm{CH}_{2}-\mathrm{C}_{6} \mathrm{H}_{4}(1,4)-4-\mathrm{SO}_{2} \mathrm{NH}_{2}$ & $-\mathrm{NH}-\mathrm{C}_{6} \mathrm{H}_{4}(1,4)-\mathrm{CH}=\mathrm{CH}-\mathrm{C}_{6} \mathrm{H}_{4}(1,4)-4-\mathrm{H}$ & 39.92 & 59.70 & 81.96 & 89.40 & 16.92 & 16.53 & 18.78 & 38.51 \\
\hline 27 & 1 & $\mathrm{NH}-\mathrm{CH}_{2} \mathrm{CH}_{2}-\mathrm{C}_{6} \mathrm{H}_{4}(1,4)-4-\mathrm{SO}_{2} \mathrm{NH}_{2}$ & $-\mathrm{NH}-\mathrm{C}_{6} \mathrm{H}_{4}(1,4)-\mathrm{CH}=\mathrm{CH}-\mathrm{C}_{6} \mathrm{H}_{4}(1,4)-3-\mathrm{OH}$ & 88.67 & 89.97 & 90.19 & 90.25 & 24.08 & 27.46 & 32.65 & 43.41 \\
\hline 28 & 1 & $\mathrm{NH}-\mathrm{CH}_{2} \mathrm{CH}_{2}-\mathrm{C}_{6} \mathrm{H}_{4}(1,4)-4-\mathrm{SO}_{2} \mathrm{NH}_{2}$ & $-\mathrm{NH}-\mathrm{C}_{6} \mathrm{H}_{4}(1,4)-\mathrm{CH}=\mathrm{CH}-\mathrm{C}_{6} \mathrm{H}_{4}(1,4)-4-\mathrm{OH}$ & 75.43 & 79.31 & 83.99 & 87.37 & 26.61 & 26.84 & 28.19 & 41.95 \\
\hline 29 & 1 & $\mathrm{NH}-\mathrm{CH}_{2} \mathrm{CH}_{2} \mathrm{CH}_{2} \mathrm{OH}$ & $-\mathrm{NH}-\mathrm{C}_{6} \mathrm{H}_{4}(1,4)-\mathrm{CH}=\mathrm{CH}-\mathrm{C}_{6} \mathrm{H}_{4}(1,4)-3-\mathrm{OH}$ & 55.92 & 65.05 & 74.47 & 82.92 & 16.69 & 21.71 & 28.98 & 39.63 \\
\hline 30 & 2 & $\mathrm{NH}-\mathrm{CH}_{2} \mathrm{CH}_{2} \mathrm{CH}_{2} \mathrm{OH}$ & $-\mathrm{NH}-\mathrm{C}_{6} \mathrm{H}_{4}(1,4)-\mathrm{CH}=\mathrm{CH}-\mathrm{C}_{6} \mathrm{H}_{4}(1,4)-3-\mathrm{OH}$ & 83.37 & 90.19 & 91.66 & 91.71 & 1.70 & 3.67 & 7.23 & 43.19 \\
\hline 31 & 2 & $\mathrm{NH}-\mathrm{CH}_{2} \mathrm{CH}_{2} \mathrm{OH}$ & $-\mathrm{NH}-\mathrm{C}_{6} \mathrm{H}_{4}(1,4)-\mathrm{CH}=\mathrm{CH}-\mathrm{C}_{6} \mathrm{H}_{4}(1,4)-4-\mathrm{OH}$ & 79.20 & 85.91 & 88.33 & 88.39 & 16.98 & 18.22 & 21.99 & 30.73 \\
\hline 32 & 2 & $\mathrm{NH}-\mathrm{CH}_{2} \mathrm{CH}(\mathrm{OH}) \mathrm{CH}_{2} \mathrm{OH}$ & $-\mathrm{NH}-\mathrm{C}_{6} \mathrm{H}_{4}(1,4)-\mathrm{CH}=\mathrm{CH}-\mathrm{C}_{6} \mathrm{H}_{4}(1,4)-3-\mathrm{OH}$ & 36.37 & 56.15 & 76.89 & 85.74 & 14.95 & 15.12 & 23.80 & 59.64 \\
\hline 33 & 2 & $\mathrm{NH}-\mathrm{CH}_{2} \mathrm{CH}(\mathrm{OH}) \mathrm{CH}_{2} \mathrm{OH}$ & $-\mathrm{NH}-\mathrm{C}_{6} \mathrm{H}_{4}(1,4)-\mathrm{CH}=\mathrm{CH}-\mathrm{C}_{6} \mathrm{H}_{4}(1,4)-4-\mathrm{OH}$ & 52.54 & 55.98 & 76.72 & 85.12 & 16.92 & 17.31 & 24.36 & 48.65 \\
\hline 34 & 2 & $\mathrm{NH}-\mathrm{C}_{6} \mathrm{H}_{4}(1,4)-4-\mathrm{OH}$ & $-\mathrm{NH}-\mathrm{C}_{6} \mathrm{H}_{4}(1,4)-\mathrm{CH}=\mathrm{CH}-\mathrm{C}_{6} \mathrm{H}_{4}(1,4)-4-\mathrm{H}$ & 90.36 & 90.31 & 90.02 & 89.85 & 18.27 & 19.34 & 27.40 & 48.20 \\
\hline
\end{tabular}




\begin{tabular}{|c|c|c|c|c|c|c|c|c|c|c|c|}
\hline 35 & 2 & $\mathrm{NH}-\mathrm{C}_{6} \mathrm{H}_{4}(1,4)-4-\mathrm{OH}$ & $-\mathrm{NH}-\mathrm{C}_{6} \mathrm{H}_{4}(1,4)-\mathrm{CH}=\mathrm{CH}-\mathrm{C}_{6} \mathrm{H}_{4}(1,4)-2-\mathrm{OH}$ & 71.03 & 75.09 & 81.96 & 87.83 & 18.78 & 19.63 & 23.68 & 44.14 \\
\hline 36 & 2 & $\mathrm{NH}-\mathrm{C}_{6} \mathrm{H}_{4}(1,4)-4-\mathrm{OH}$ & $-\mathrm{NH}-\mathrm{C}_{6} \mathrm{H}_{4}(1,4)-\mathrm{CH}=\mathrm{CH}-\mathrm{C}_{6} \mathrm{H}_{4}(1,4)-3-\mathrm{OH}$ & 90.08 & 89.91 & 89.80 & 89.57 & 28.53 & 31.97 & 34.28 & 48.26 \\
\hline 37 & 2 & $\mathrm{NH}-\mathrm{C}_{6} \mathrm{H}_{4}(1,4)-4-\mathrm{OH}$ & $-\mathrm{NH}-\mathrm{C}_{6} \mathrm{H}_{4}(1,4)-\mathrm{CH}=\mathrm{CH}-\mathrm{C}_{6} \mathrm{H}_{4}(1,4)-4-\mathrm{OH}$ & 91.04 & 90.98 & 90.81 & 90.64 & 23.51 & 25.21 & 32.31 & 29.55 \\
\hline Trolox & - & - & - & 89.29 & 89.23 & 88.18 & 89.23 & 34.96 & 35.41 & 37.61 & 41.49 \\
\hline Ascorbic acid & - & - & - & 88.33 & 88.45 & 88.33 & 88.45 & 29.09 & 28.81 & 29.83 & 31.07 \\
\hline
\end{tabular}

${ }^{1}$ Compounds previously reported in $[16] ;{ }^{2}$ Errors in the range of $1-5 \%$ of the reported value (Mean from three different assays). 
Over twenty derivatives fully captured the cation radical $\mathrm{ABTS}^{+}$after a few seconds at the highest used concentration of tested compounds $1 \times 10^{-2} \mathrm{M}$. At the lowest concentration of $1 \times 10^{-4} \mathrm{M}$ at time $0 \mathrm{~min}$, for ten derivatives was \%inhibition of the cation radical $\mathrm{ABTS}^{+}$comparable or better than both used standards (Trolox and ascorbic acid). Furthermore, derivatives 1, 2, 9, 24, and 25 showed after $60 \mathrm{~min}$ almost double \%inhibition activity (73.44-87.09\%) in comparison to standard Trolox (41.09\%) and ascorbic acid (31.07\%).

From the results presented in Table 2, some general statements can be made. Compounds with the highest values of \%inhibition activity contain the phenolic fragment in their structure. The hydroxy group can inhibit (scavenge) the cation radical $\mathrm{ABTS}^{+}$probably by the single-electron transfer mechanism (SET). The resulting cation radical formed after scavenging is stabilized by the delocalization of the electron across the molecule. The presence of the oxo group $(\mathrm{C}=\mathrm{O})$ and the unsaturated double bond in the chalcone structural motif enhanced the antioxidative properties of the phenolic hydroxy group. This is caused by a very high electron delocalization of the formed cation radical, which is supported by the electron-withdrawing character of the carbonyl group. In fact, the compounds containing the hydroxychalcone moieties are, in general, the most active of tested compounds. On the other hand, the compounds incorporating the stilbene structural motif exhibit lower \%inhibition of the cation radical $\mathrm{ABTS}^{+}$. The reason is less effective conjugation of the cation radical in comparison with the chalcone derivatives. In general, a higher level of electron delocalization of formed cation radical increases the energy of HOMO orbital, which means the better oxidation-reduction potential of the compound and, therefore, the better oxidative activity.

The ability of the tested compounds to donate the hydrogen proton also effects the \%inhibition of the cation radical ABTS ${ }^{+}$. Compounds containing the hydroxychalcone structural moiety, which is more willing to donate the hydrogen proton, showed significantly better antioxidative activity than compounds containing hydroxystilbene structural motif, which has a much lower ability to donate the hydrogen proton.

Table 2. ABTS cation radical scavenging ability of the most active compounds (the highest \%inhibition) expressed as $\mathrm{IC}_{50}(\mu \mathrm{M})$ in times $0 \mathrm{~min}$ and $60 \mathrm{~min}$.

\begin{tabular}{|c|c|c|c|c|c|}
\hline \multirow{2}{*}{ Compound } & \multirow{2}{*}{$\mathbf{n}$} & \multirow{2}{*}{$\mathbf{R}_{1}$} & \multirow{2}{*}{$\mathbf{R}_{2}$} & \multicolumn{2}{|c|}{$\mathrm{IC}_{50}(\mu \mathrm{M})^{2}$} \\
\hline & & & & $0 \mathrm{~min}$ & $60 \mathrm{~min}$ \\
\hline $\mathbf{1}^{1}$ & 1 & $\mathrm{NH}-\mathrm{C}_{6} \mathrm{H}_{4}(1,4)-4-\mathrm{OH}$ & $\mathrm{Cl}$ & 103.89 & 26.59 \\
\hline $2^{1}$ & 2 & $\mathrm{NH}-\mathrm{C}_{6} \mathrm{H}_{4}(1,4)-4-\mathrm{OH}$ & $\mathrm{Cl}$ & 107.20 & 17.16 \\
\hline $7^{1}$ & 1 & {$\left[\mathrm{~N}\left(\mathrm{CH}_{2} \mathrm{CH}_{2}\right)_{2} \mathrm{~N}\right] \mathrm{CH}_{2} \mathrm{COOCH}_{3}$} & $\mathrm{NH}-\mathrm{CH}_{2} \mathrm{CH}_{2}-\mathrm{C}_{6} \mathrm{H}_{4}(1,4)-4-\mathrm{SO}_{2} \mathrm{NH}_{2}$ & 317.78 & 43.84 \\
\hline $8^{1}$ & 1 & {$\left[\mathrm{~N}\left(\mathrm{CH}_{2} \mathrm{CH}_{2}\right)_{2} \mathrm{~N}\right] \mathrm{CH}_{2} \mathrm{CH}_{2} \mathrm{COOCH}_{3}$} & $\mathrm{NH}-\mathrm{C}_{6} \mathrm{H}_{4}(1,4)-4-\mathrm{OH}$ & 180.98 & 51.47 \\
\hline $9^{1}$ & 1 & $\mathrm{NH}-\mathrm{C}_{6} \mathrm{H}_{4}(1,4)-4-\mathrm{OH}$ & $\mathrm{NH}-\mathrm{CH}_{2} \mathrm{CH}(\mathrm{OH}) \mathrm{CH}_{2} \mathrm{OH}$ & 127.98 & 27.78 \\
\hline 18 & 2 & $\mathrm{NH}-\mathrm{CH}_{2} \mathrm{CH}_{2} \mathrm{OH}$ & $\begin{array}{c}-\mathrm{NH}-\mathrm{C}_{6} \mathrm{H}_{4}(1,4)-(\mathrm{C}=\mathrm{O})-\mathrm{CH}=\mathrm{CH}- \\
\mathrm{C}_{6} \mathrm{H}_{4}(1,4)-2-\mathrm{OH}\end{array}$ & 1800.00 & 153.70 \\
\hline 19 & 2 & $\mathrm{NH}-\mathrm{CH}_{2} \mathrm{CH}_{2} \mathrm{OH}$ & $\begin{array}{c}-\mathrm{NH}-\mathrm{C}_{6} \mathrm{H}_{4}(1,4)-(\mathrm{C}=\mathrm{O})-\mathrm{CH}=\mathrm{CH}- \\
\mathrm{C}_{6} \mathrm{H}_{4}(1,4)-3-\mathrm{OH}\end{array}$ & 288.06 & 20.16 \\
\hline 24 & 2 & $\mathrm{NH}-\mathrm{C}_{6} \mathrm{H}_{4}(1,4)-4-\mathrm{OH}$ & $\begin{array}{c}-\mathrm{NH}-\mathrm{C}_{6} \mathrm{H}_{4}(1,4)-(\mathrm{C}=\mathrm{O})-\mathrm{CH}=\mathrm{CH}- \\
\mathrm{C}_{6} \mathrm{H}_{4}(1,4)-2-\mathrm{OH}\end{array}$ & 147.43 & 45.78 \\
\hline 25 & 2 & {$\left[\mathrm{~N}\left(\mathrm{CH}_{2} \mathrm{CH}_{2}\right)_{2} \mathrm{~N}\right] \mathrm{CH}_{2} \mathrm{CH}_{2} \mathrm{COOCH}_{3}$} & $\begin{array}{c}-\mathrm{NH}-\mathrm{C}_{6} \mathrm{H}_{4}(1,4)-(\mathrm{C}=\mathrm{O})-\mathrm{CH}=\mathrm{CH}- \\
\mathrm{C}_{6} \mathrm{H}_{4}(1,4)-4-\mathrm{OH}\end{array}$ & 53.72 & 17.22 \\
\hline Trolox & - & - & - & 293.46 & 178.33 \\
\hline Ascorbic acid & - & - & - & 169.24 & 147.47 \\
\hline
\end{tabular}

${ }^{1}$ Compounds previously reported in [16]. ${ }^{2}$ Errors in the range of $1-5 \%$ of the reported value (Mean from three different assays).

The numbers of IC 50 were obtained as described in the experimental section. The values of IC 50 correlated with the results of the determination of the \%inhibition of cation radical $\mathrm{ABTS}^{+}$. From results presented in Table 2, it is clear that IC 50 values of the most active compounds after $60 \mathrm{~min}$ (IC $\mathrm{I}_{50}$ $=17.16-79.34 \mu \mathrm{M}$ ) were nearly ten times lower than IC 50 values of both used standards (Trolox IC $50=$ 178.33; ascorbic acid IC $50=147.47)$. The structure of the three derivatives with the highest antioxidant activity (the lowest values of $\mathrm{IC}_{50}$ ) is very diverse: compound 2 is a disubstituted derivative containing aminobenzenesulfonamide and aminophenol structural motive. Hit 19 contains aminobenzenesulfonamide, aminoethanol, and 3-hydroxy-aminochalcone structural fragments, and 
compound 25 contains aminobenzenesulfonamide, piperazine, and 4-hydroxy-chalcone motive. All three compounds have the common structural fragment, phenolic hydroxyl group, which is probably responsible for their antioxidative activity.

Tested compounds showed great potential as antioxidants useful in the treatment of diseases caused by oxidative stress. Besides, the antioxidant effect is a great benefit for compounds that are excellent inhibitors of hCA IX (compound 1) [16] since oxidative stress is associated with tumor development and progression in several tumors. Also, the antioxidative properties may have a beneficial effect in suppressing the side effects associated with the treatment using some classic cytostatics $[17,18]$.

\section{Conclusions}

In this paper, a series of 1,3,5-triazine derivatives, incorporating aminobenzenesulfonamide, aminoalcohol/ phenol, piperazine, chalcone, or stilbene structural motifs were evaluated as potential antioxidants. Target compounds were prepared by using step by step nucleophilic substitution of chlorine atoms in starting 2,4,6-trichloro-1,3,5-triazine in the presence of anhydrous potassium carbonate. $\mathrm{Cu}(\mathrm{I})$-supported on a weakly acidic resin catalyzed the reaction. The antioxidant activity was determined in terms of \%inhibition activity and IC 50 , using the ABTS method. Trolox and ascorbic acid were used as standards. At the lowest concentration $1 \times 10^{-4} \mathrm{M}$ at time $0 \mathrm{~min}$, \%inhibition of the cation radical $\mathrm{ABTS}^{+}$was comparable or better than both used standards for ten derivatives. Furthermore, after 60 min compounds 1, 2, 9 and 25 showed nearly twice \%inhibition (73.44-87.09\%) in comparison with standards (Trolox $=41.49 \%$; ASA $=31.07 \%$ ). Values of $\mathrm{IC}_{50}$ correlated with \%inhibition activity. For compounds 1, 2, 9 and 25 values of IC 50 in time $60 \mathrm{~min}(17.16-27.78 \mu \mathrm{M})$ were 5 times lower than $\mathrm{IC}_{50}$ of both standards (Trolox $=178.33 \mu \mathrm{M}$; ASA $=147.47 \mu \mathrm{M}$ ). From results, it is clear that more than half of the tested compounds showed great potential as antioxidants useful in the treatment of diseases caused by oxidative stress.

Author Contributions: Methodology, E.H., P.P. and J.C.; validation, E.H., T.P. and N.Č.; data curation, E.H., T.P., N.Č.; writing - original draft preparation, E.H., T.P., N.Č.; writing - review and editing, P.P., J.C. and R.O.; supervision, P.P., J.C. and R.O.; project administration, E.H. and T.P.; funding acquisition, N.Č. and O.P. All authors have read and agreed to the published version of the manuscript.

Funding: This research was funded by the Internal grant agency of the University of Veterinary and Pharmaceutical Sciences Brno, grant number 317/2017/FaF, and by Institutional support for research of the Masaryk university Brno (MUNI 2222/312).

Conflicts of Interest: The authors declare no conflict of interest. The funders had no role in the design of the study; in the collection, analyses, or interpretation of data; in the writing of the manuscript, or in the decision to publish the results.

\section{References}

1. Pisoschi, A.M.; Pop, A. The role of antioxidants in the chemistry of oxidative stress: A review. Eur. J. Med. Chem. 2015, 97, 55-74.

2. Persson, T.; Popescu, B.O.; Cedazo-Minguez, A. Oxidative Stress in Alzheimer's Disease: Why Did Antioxidant Therapy Fail? Oxidative Med. Cell. Longev. 2014, 2014, doi:10.1155/2014/427318.

3. Gonzalez, P.; Pota, K.; Turan, L.S.; da Costa, V.C.P.; Akkaraju, G.; Green, K.N. Synthesis, Characterization, and Activity of a Triazine Bridged Antioxidant Small Molecule. ACS Chem. Neurosci. 2017, 8, 2414-2423.

4. Neha, K.; Haider, M.R.; Pathak, A.; Yar, M.S. Medicinal prospects of antioxidants: A review. Eur. J. Med. Chem. 2019, 178, 687-704.

5. Sisein, E.A. Biochemistry of free radicals and antioxidants. Sch. Acad. J. Biosci. 2014, 2, 110-118.

6. Kumar, S. The importance of antioxidant and their role in pharmaceutical science-A review. Asian. J. Med. Chem. Pharm. Sci. 2014, 1, 27-44.

7. López-Alarcón, C.; Denicola, A. Evaluating the antioxidant capacity of natural products: A review on chemical and cellular-based assays. Anal. Chim. Acta 2013, 763, 1-10. 
8. Maulik, N.; McFadden, D.; Otani, H.; Thirunavukkarasu, M.; Parinandi, N.L. Antioxidants in Longevity and Medicine. Oxidative Med. Cell. Longev. 2013, 2013, doi:10.1155/2013/82067.

9. Toda, S. Polyphenol Content and Antioxidant Effects in Herb Teas. Chin. Med. 2011, 2, $29-31$.

10. Tripathi, P.N.; Srivastava, P.; Sharma, P.; Tripathi, M.K.; Seth, A.; Tripathi, A.; Rai, S.N.; Singh, S.P.; Shrivastava, S.K. Biphenyl-3-oxo-1,2,4-triazine linked piperazine derivatives as potential cholinesterase inhibitors with anti-oxidant property to improve the learning and memory. Bioorg. Chem. 2019, 85, 82-96.

11. Iraji, A.; Firuzi, O.; Khoshneviszadeh, M.; Nadri, H.; Edraki, N.; Miri, R. Synthesis and structure-activity relationship study of multi-target triazine derivatives as innovative candidates for treatment of Alzheimer's disease. Bioorg. Chem. 2018, 77, 223-235.

12. Akocak, S.; Boga, M.; Lolak, N.; Tuneg, M.; Sanku, R.K.K. Design, synthesis and biological evaluation of 1,3-diaryltriazene-substituted sulfonamides as antioxidant, acetylcholinesterase and butyrylcholinesterase inhibitors. J. Turk. Chem. Soc. Sect. A Chem. 2019, 6, 63-70.

13. Narsinghani, T.; Sharma, M.C.; Bhargav, S. Synthesis, docking studies and antioxidant activity of some chalcone and aurone derivatives. Med. Chem. Res. 2013, 22, 4059-4068.

14. San Miguel-Chávez, R. Phenolic Antioxidant Capacity: A Review of the State of the Art. In Phenolic Compounds-Biological Activity; IntechOpen: London, UK, 2017; pp. 59-74.

15. Havránková, E.; Csöllei, J.; Pazdera, P. New Approach for the One-Pot Synthesis of 1,3,5-Triazine Derivatives: Application of $\mathrm{Cu}(\mathrm{I})$ Supported on a Weakly Acidic Cation-Exchanger Resin in a Comparative Study. Molecules 2019, 24, 3586.

16. Havránková, E.; Csöllei, J.; Vullo, D.; Garaj, V.; Pazdera, P.; Supuran, C.T. Novel sulfonamide incorporating piperazine, aminoalcohol and 1,3,5-triazine structural motifs with carbonic anhydrase I, II and IX inhibitory action. Bioorg. Chem. 2018, 77, 25-37.

17. Singh, K.; Bhori, M.; Kasu, Y.A.; Bhat, G.; Marar, T. Antioxidants as precision weapons in war against cancer chemotherapy induced toxicity -Exploring the armoury of obscurity. Saudi Pharm. J. 2018, 26, 177-190.

18. Mut-Salud, N.; Álvarez, P.J.; Garrido, J.M.; Carrasco, E.; Aránega, A.; Rodríguez-Serrano, F. Antioxidant Intake and Antitumor Therapy: Toward Nutritional Recommendations for Optimal Results. Oxidative Med. Cell. Longev. 2016, 2016, doi:10.1155/2016/6719534.

(C) 2019 by the authors. Licensee MDPI, Basel, Switzerland. This article is an open access article distributed under the terms and conditions of the Creative Commons Attribution (CC BY) license (http://creativecommons.org/licenses/by/4.0/). 